\title{
Capacidade de atendimento hospitalar mediante solicitações de leitos de Unidade de Terapia Intensiva adulto
}

\author{
Hospital service capacity assessment through requesting adult Intensive Care Unint beds \\ Capacidad de servicio hospitalario a través solicitudes de camas de Unidad de Cuidados Intensivos
} para adultos

Recebido: 27/05/2021 | Revisado: 04/06/2021 | Aceito: 21/06/2021 | Publicado: 04/07/2021

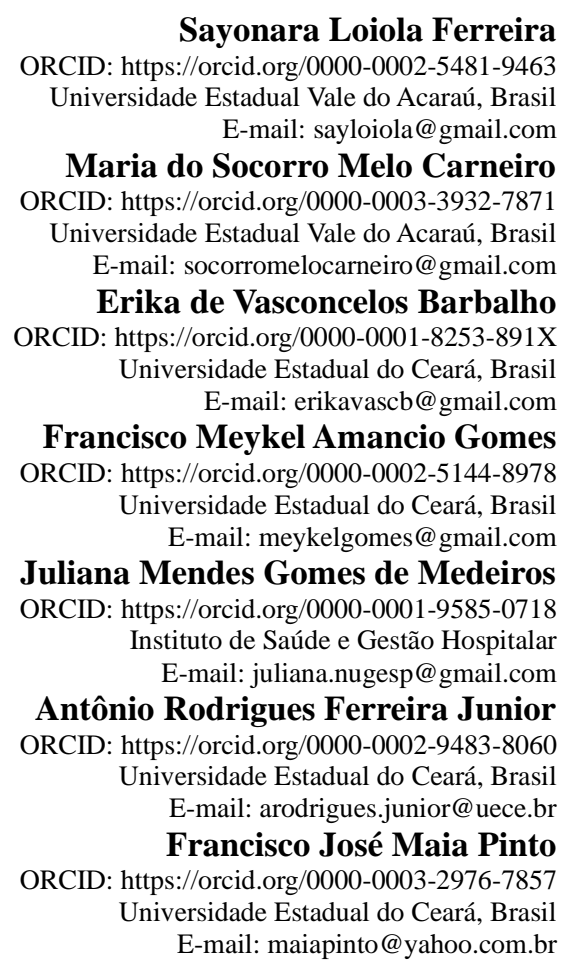

\section{Resumo}

Objetivo: analisar a capacidade de atendimento hospitalar mediante solicitações de leitos de UTI, em hospital de referência. Método: estudo transversal, realizado em um hospital situado no município de Sobral-CE, de janeiro a março de 2019. A amostra correspondeu a 489 pacientes. Considerou-se como desfecho capacidade de atendimento, quanto à disponibilidade de leitos de UTI, dicotomizado em não atendido e atendido. Como variáveis associativas: sexo, idade, procedência, diagnóstico, prioridade, quantidade de solicitações, tempo de espera e situação de atendimento. Empregou-se o teste de Qui-quadrado de Wald e a regressão múltipla para os modelos não ajustado e ajustado, respectivamente. Resultados: a capacidade de atendimento esteve associada com: idade, prioridade, diagnóstico clínico, situação de atendimento e tempo de espera. Conclusão: a capacidade de atendimento foi restrita na resolução dos problemas da população do município, sede e adjacências, em relação à terapia intensiva, indicando baixa oferta de leitos em relação à demanda de solicitações.

Palavras-chave: Administração de serviços de saúde; Número de leitos em hospital; Unidades de terapia intensiva.

\begin{abstract}
Objective: To analyze the hospital care capacity through requesting ICU beds in a reference hospital. Method: crosssectional study, carried out at in a hospital located in the municipality of Sobral-CE, from January to march 2019. The sample was 489 patients. The primary outcome was the attendance capacity, regarding the availability of ICU beds, dichotomized as unattended and attended. Associative variables analyzed were: sex, age, origin, diagnosis, priority, number of requests, waiting time and service situation. The Wald chi-square test and multiple regression were used for data analysis. Results: a significant association was identified with between primary outcome and age, priority, clinical diagnosis, service situation and waiting time. Conclusion: the service capacity was restricted to solving the
\end{abstract}


problems of the municipality, headquarters and surroundings population, in relation to intensive care, and results indicated a low supply of beds in relation to the demand for requests.

Keywords: Health services administration; Hospital bed capacity; Intensive care units.

\section{Resumen}

Objetivo: analizar la capacidad de atención hospitalaria a través de solicitudes de camas de UCI, en un hospital de referencia. Método: estudio transversal, realizado en un hospital ubicado en el municipio de Sobral-CE, de enero a marzo de 2019. La muestra correspondió a 489 pacientes. El resultado fue la capacidad de asistencia, en cuanto a la disponibilidad de camas de UCI, dicotomizada como desatendida y atendida. Como variables asociativas: sexo, edad, procedencia, diagnóstico, prioridad, número de solicitudes, tiempo de espera y situación del servicio. Se utilizaron la prueba de chi-cuadrado de Wald y la regresión múltiple para los modelos ajustados y no ajustados, respectivamente. Resultados: la capacidad asistencial se asoció con: edad, prioridad, diagnóstico clínico, situación asistencial y tiempo de espera. Conclusión: la capacidad del servicio se vio restringida en la solución de los problemas de la población del municipio, sede y alrededores, en relación a cuidados intensivos, lo que indica una baja oferta de camas en relación a la demanda de solicitudes.

Palabras clave: Administración de los servicios de salud; Capacidad de camas en hospitales; Unidades de cuidados intensivos.

\section{Introdução}

A Unidade de Terapia Intensiva (UTI) é uma instalação presente nos equipamentos de saúde que dispõe de recursos materiais e humanos de suporte avançado, apropriados a fornecer cuidados intensivos aos pacientes em estado crítico. Essas unidades variam conforme o foco do atendimento, podendo ser gerais ou mistas, clínicas e/ou cirúrgicas ou concentrar sua assistência em um subgrupo de pacientes, variando pela assistência aos sistemas, patologias ou problemas específicos e também por grupos de idade (Nates et al., 2016; Brasil, 2020).

No Brasil, em dezembro de 2019 havia 46.045 leitos de UTI, sendo 23.049 do Sistema Único de Saúde (SUS) e 22.996 na rede privada. Em abril de 2020, com mobilização para ampliação destes em decorrência da pandemia pelo novo coronavírus, o número foi ampliado para 60.265, sendo 26.153 leitos SUS e 34.112 privados, mostrando predominância no avanço do setor privado. A região Sudeste concentra o maior número de leitos, com 51,9\% do total, seguida da região nordeste, com $20,7 \%$. No entanto, quando se analisa por região, a relação de leitos de UTI do SUS por habitante, as iniquidades são bem claras, onde a região nordeste passa a ser a segunda com menor número de leitos públicos de UTI por habitante. Vale ressaltar que nesta região 88,43\% da população é dependente do SUS e que, diante de 5.968 leitos públicos de UTI, a relação passa a ser de aproximadamente um leito a cada 8.456 pessoas (Cotrim Junior \& Cabral, 2020). Esta configuração pressupõe, portanto, uma insuficiência de leitos de UTI diante das solicitações (Chagas, Vilardo e Noronha, 2019; Alves Júnior, Passos e AlmeidaSantos, 2020).

Este descompasso entre a fila de espera e a solicitação de leitos possui causas inter-relacionadas que depende do: perfil demográfico e epidemiológico, referente a demanda, capacidade instalada de leitos e mecanismos de gestão. Além disso, há fatores: técnicos e humanos, governança clínica e regulação, que determinarão o tempo de permanência no leito e os fluxos de alta (Goldwasser et al., 2018). Diante da complexidade dos quadros clínicos dos pacientes com solicitação de vaga de UTI, associada à situação crítica de disponibilidade destas, as admissões precisam ser baseadas em critérios específicos, como o diagnóstico e o potencial benéfico das intervenções terapêuticas (Chagas, Vilardo e Noronha, 2019; Alves Júnior, Passos; Almeida-Santos, 2020).

Considerando-se que os custos do sistema de saúde são maiores, quanto maior for a demanda por leito de terapia intensiva e mediante a escassez de recursos disponíveis, exige-se adequada utilização e admissão fortemente resguardada destes leitos (Nates et al., 2016).

Neste sentido, justifica-se a análise detalhada deste contexto para responder às inquietações quanto ao atendimento das demandas por leitos de UTI, o destino das solicitações que não obtiveram sucesso e os desfechos de pacientes após a 
ocupação dos leitos. Assim, pretende-se, contribuir com a compreensão desta problemática que tanto afeta os diferentes contextos da saúde pública brasileira.

Este estudo objetivou analisar a capacidade de atendimento hospitalar mediante solicitações de leitos de UTI adulto, de um hospital terciário de referência.

\section{Metodologia}

Trata-se de uma pesquisa transversal, com abordagem descritiva e analítica, realizada em um hospital, situado no município de Sobral-CE, no período de janeiro a março de 2019.

O referido hospital é o maior serviço de alta complexidade do interior do Norte e Nordeste, com 57. 813,70 metros quadrados de área construída. É referência para cerca de 1,6 milhão de habitantes dos municípios das cinco Áreas Descentralizadas de Saúde (ADS) da Região de Saúde de Sobral. Tem capacidade instalada de 30 leitos de UTI Adulto e Pediátrica, 17 salas cirúrgicas e 269 leitos de internação. Na assistência à saúde da mulher disponibiliza 40 leitos de UTI e Intermediária Neonatal, além de oferecer outros serviços especializados (ISGH, 2017).

A população envolveu os pacientes adultos internados que tiveram solicitação de leito de UTI, totalizando 1335 solicitações. Foram excluídas as solicitações com dados incompletos. A amostra final foi obtida por conveniência correspondendo a 489 pacientes.

Nesta pesquisa foi considerado como desfecho a capacidade de atendimento quanto à disponibilidade de leitos da UTI, dicotomizado em não atendido e atendido. Consideraram-se as seguintes variáveis associativas:

Sexo: corresponde à classificação quanto ao gênero, sendo o masculino o exposto.

Idade (anos): classificada em acima de 65 anos e até 65 anos. A escolha dessas faixas etárias foi baseada no estudo de Angus et al. e Capela et al.

Procedência: correspondeu às ADS do paciente, sendo: procedência 1 - ADS 1 (Acaraú), procedência 2 - ADS 2 (Camocim), procedência 3 - ADS 3 (Crateús), procedência 4 - ADS 5 (Tianguá) e procedência 5 - ADS 4 (Sobral) (Ceará, 2019).

Diagnóstico: refere-se ao sistema orgânico acometido, com necessidade de leito de UTI.

Prioridade de atendimento: a regulação dos leitos de UTI se baseia em um critério técnico de prioridades de atendimento, seguindo protocolos hierarquizados, construídos com base em consensos de sociedades médicas especializadas, e dando prioridade aos pacientes com doença mais grave (Goldwasswer et al., 2018). Neste estudo, os pacientes com solicitação de vaga para UTI foram classificados em quatro grupos distintos (prioridades P1, P2, P3 e P4), de acordo com critérios de prioridades de admissão em UTI, conforme a Portaria no 2.156/2016 (CFM, 2016). As prioridades foram: P1 - pacientes gravemente doentes, instáveis, necessitando de monitorização e tratamento intensivo, com chances significativas de recuperação; P2 - necessidade de monitorização intensiva pela possibilidade de descompensação e de intervenção imediata; P3 - pacientes criticamente doentes, com baixa probabilidade de recuperação por conta da gravidade da doença aguda ou presença de comorbidades; P4 - pacientes com pouco ou nenhum benefício com a internação na UTI e P5 - Pacientes com doença em fase de terminalidade, ou moribundos, sem possibilidade de recuperação.

Situação de atendimento: classificou-se em óbito (os pacientes que faleceram ao solicitar/conseguir a vaga na UTI) e alta/transferência (aqueles que tiveram sua condição patológica resolvida ou foram transferidos para outro setor ou instituição).

Quantidade de solicitações de leito de UTI: permite estabelecer uma previsão futura da demanda (fluxo de admissão). O retardo na disponibilidade de leitos resulta em impacto negativo no desfecho clínico dos pacientes e na taxa de mortalidade (Santos, Machado e Lobo, 2020). Levando em consideração que, no hospital estudado, cada solicitação é atualizada a cada 24 
horas (Portaria $\mathrm{n}^{\circ}$ 2.156/2016, CFM, 2016) ou pela prioridade de atendimento, considerou-se os pacientes dicotomizado em, até duas solicitações e os que tiveram a partir de duas solicitações.

Tempo de espera pelo leito de UTI: pode sinalizar a relação existente entre alguns fatores envolvidos no processo de gestão e de regulação, possibilitando observar a projeção futura de demanda. Neste estudo considerou-se o tempo acima de seis horas e de até seis horas de espera. Uma espera de seis horas é considerada clinicamente viável para manter o prognóstico inalterado, caso o paciente receba medidas de suporte pré-hospitalares e emergenciais adequadas. É uma variável que pode sofrer influência da quantidade de solicitações, da oferta de leitos e da prioridade de atendimento (Goldwasser et al., 2016).

Para coleta de dados foi realizada análise documental dos relatórios de solicitações de ocupação dos leitos da UTI adulto do hospital referido e do sistema interno da instituição. Como instrumento, foi utilizado um formulário estruturado para verificar as variáveis das solicitações de leitos que foram estudadas.

Os dados foram armazenados no programa Excel e o processamento realizado no programa SPSS (Statistical Package for the Social Sciences, San Diego, Califórnia, Estados Unidos da América) versão 23.0.

$\mathrm{Na}$ análise descritiva, os dados foram interpretados por meio das frequências (absoluta e percentual). Na análise bivariada, não ajustada foi verificada a existência de associação, entre o desfecho e as variáveis associativas, empregando-se o teste de Qui-quadrado de Wald, ao nível de significância de 5\%. Utilizou-se a regressão de Poisson com variância robusta, como estimativa por ponto e por intervalo, da Razão de Prevalência (RP), que produz estimativas adequadas e intervalos de confiança independente da prevalência adotada (Barros \& Hirakata, 2013; Reis et al., 2010). Considerou-se como critério de entrada para o ajustamento do modelo, as variáveis que apresentaram valor descritivo $p<0,20$ e para permanecer no modelo final ajustado, apenas, aquelas que indicaram valor descritivo $\mathrm{p}<0,05$. Foi estimada a RP, para quantificar as associações significativas, com intervalo de confiança de $95 \%$.

A pesquisa foi aprovada pelo Comitê de Ética em Pesquisa da Universidade Estadual Vale do Acaraú, sob protocolo $\mathrm{N}^{\circ} 3.195 .679$.

\section{Resultados e Discussão}

Observou-se que dentre os 489 pacientes investigados, a maioria: tinha a partir de 65 anos 275 (56, 2\%), era do sexo masculino 258 (52,8\%), procedente dos municípios da Área Descentralizada de Saúde 4 (município de Sobral e adjacências) 267 (54,6\%) e, não conseguiu a vaga de UTI requerida 303 (62\%).

Clinicamente, dentre as comorbidades apresentadas para os pedidos de vagas de UTI, as doenças respiratórias foram as mais frequentes $157(32,1 \%)$, ligeiramente abaixo das demais doenças correspondentes as outras partes do corpo 167 (34, $2 \%)$, e seguida pelas que acometeram os sistemas digestivo 88 (18\%) e cardiovascular 77 (15,7\%).

A fim de caracterizar a demanda (alta, média e baixa) por leitos de UTI, foram analisadas as variáveis: prioridade de atendimento (gravidade clínica), quantidade de vezes em que houve solicitação de leito para o mesmo paciente e tempo de espera pela vaga. Deste modo, as variáveis que tiveram maior ocorrência de solicitações foram: prioridade 1 (P1) 209 (42,7\%), tempo de espera acima de seis horas $348(71,2 \%)$ e quantidade de até duas solicitações $320(65,4 \%)$. No tocante a situação de atendimento, foram a óbito 294 pacientes $(60,1)$.

No modelo não ajustado, ao relacionar a variável desfecho com as variáveis associativas, foram consideradas para o modelo final aquelas com $\mathrm{p}<0,20$, sendo: idade $(<0,001)$, sexo $(0,098)$, diagnóstico clínico $(<0,002)$, prioridade de atendimento $(<0,001)$, tempo de espera $(<0,003)$, quantidade de solicitações $(<0,001)$ e situação de atendimento $(<0,001)$. Apenas a variável procedência não foi inclusa no modelo final $(\mathrm{p}=0,681)$ (Tabela 1$)$. 
Na análise multivariada, por meio da regressão de Poisson, identificou-se associação significativa para as variáveis: idade $(<0,001)$ prioridade $(<0,001)$, diagnóstico clínico $(0,027)$, situação de atendimento $(0,013)$ e tempo de espera $(<0,002)$ (Tabela 2).

Tabela 1 - Relação no modelo não ajustado, entre o desfecho e as variáveis explanatórias, com o valor de p. Sobral, Ceará, 2019

\begin{tabular}{|c|c|c|c|c|c|c|c|}
\hline \multirow{3}{*}{ Variáveis } & \multicolumn{4}{|c|}{ Capacidade de atendimento } & \multirow[b]{3}{*}{$\mathbf{R P}$} & \multirow[b]{3}{*}{ IC $(95 \%)$} & \multirow[b]{3}{*}{ p } \\
\hline & \multirow{2}{*}{$\begin{array}{c}\text { Não } \\
\text { resolvido } \\
\text { n } \\
\end{array}$} & \multicolumn{3}{|c|}{ Resolvido } & & & \\
\hline & & $\%$ & $\mathbf{n}$ & $\%$ & & & \\
\hline \multicolumn{8}{|l|}{ Idade } \\
\hline A partir de 65 anos & 209 & 69 & 66 & 35,5 & 1,73 & 1,$46 ; 2,04$ & $<0,001$ \\
\hline Abaixo de 65 anos & 94 & 31 & 120 & 64,5 & 1 & & \\
\hline \multicolumn{8}{|l|}{ Sexo } \\
\hline Masculino & 151 & 49,8 & 107 & 57,5 & 0,88 & 0,$77 ; 1,02$ & 0,098 \\
\hline Feminino & 152 & 50,2 & 79 & 42,5 & 1 & & \\
\hline Procedência & & & & & & & 0,681 \\
\hline ADS 1 & 30 & 9,9 & 21 & 11,3 & 1,05 & 0,$77 ; 1,42$ & \\
\hline ADS 2 & 28 & 9,2 & 19 & 10,2 & 1,06 & 0,$78 ; 1,45$ & \\
\hline ADS 3 & 33 & 10,9 & 16 & 8,6 & 1,20 & 0,$90 ; 1,59$ & \\
\hline ADS 4 & 170 & 56,1 & 97 & 52,2 & 1 & & \\
\hline ADS 5 & 42 & 13,9 & 33 & 17,7 & 1,13 & 0,$91 ; 1,41$ & \\
\hline \multicolumn{7}{|l|}{ Diagnóstico classificação } & $<0,002$ \\
\hline Doenças cardiovasculares & 48 & 15,8 & 29 & 15,6 & 1,23 & 0,$98 ; 1,56$ & \\
\hline Doenças respiratórias & 107 & 35,3 & 50 & 26,9 & 1,35 & 1,$12 ; 1,63$ & \\
\hline Doenças do aparelho digestivo & 64 & 21,1 & 24 & 12,9 & 1,446 & 1,$18 ; 1,76$ & \\
\hline Outras doenças & 84 & 27,7 & 83 & 44,6 & 1 & & \\
\hline Prioridade & & & & & & & $<0,001$ \\
\hline Prioridade 1 & 91 & 30 & 118 & 63,4 & 0,48 & 0,$39 ; 0,58$ & \\
\hline Prioridade 2 & 90 & 29,7 & 47 & 25,3 & 0,72 & 0,$61 ; 0,86$ & \\
\hline Prioridade 3 & 94 & 31 & 18 & 9,7 & 0,92 & 0,$80 ; 1,07$ & \\
\hline Prioridade 4 & 28 & 9,2 & 3 & 1,6 & 1 & & \\
\hline \multicolumn{8}{|l|}{ Tempo de espera } \\
\hline Acima de 6 horas & 201 & 66,3 & 147 & 79 & 0,79 & 0,$69 ; 0,91$ & $<0,003$ \\
\hline Até 6 horas & 102 & 33,7 & 39 & 21 & 1 & & \\
\hline \multicolumn{8}{|l|}{ Quantidade de solicitações } \\
\hline Acima de 2 solicitações & 122 & 40,3 & 47 & 25,3 & 1,27 & 1,$11 ; 1,45$ & $<0,001$ \\
\hline Até 2 solicitações & 181 & 59,7 & 139 & 74,7 & 1 & & \\
\hline \multicolumn{8}{|l|}{ Situação de atendimento } \\
\hline Alta/transferência & 94 & 31 & 101 & 54,3 & 0,68 & 0,$57 ; 0,79$ & $<0,001$ \\
\hline Óbito & 209 & 69 & 85 & 45,7 & 1 & & \\
\hline
\end{tabular}

RP: Razão de Prevalência; IC 95\%:Intervalo de Confiança de 95\%.

p: teste de associação usando o Qui-quadrado de Wald. Fonte: autores. 
Tabela 2 - Modelo final ajustado entre a capacidade de atendimento e as variáveis associativas. Sobral, Ceará, 2019.

\begin{tabular}{lccc}
\hline Variável & $\mathrm{RP}$ & $\mathrm{IC} 95 \%$ & $\mathrm{p}$ \\
\hline Idade & & & $<\mathbf{0 , 0 0 1}$ \\
A partir de 65 anos & 1,45 & 1,$23 ; 1,71$ & \\
Abaixo de 65 anos & 1 & & $\mathbf{0 , 0 2 7}$ \\
& & & 0,097 \\
Diagnóstico classificação & 1,19 & 0,$96 ; 1,47$ & 0,020 \\
Doenças cardiovasculares & 1,21 & 1,$03 ; 1,43$ & 0,003 \\
Doenças respiratórias & 1,31 & 1,$09 ; 1,58$ & \\
Doenças do aparelho digestivo & 1 & & $<\mathbf{0 , 0 0 1}$ \\
Outras doenças & & & 0,001 \\
& & & 0,53 \\
Prioridade & 0,62 & 0,$50 ; 0,76$ & 0,98 \\
Prioridade 1 & 0,83 & 0,$69 ; 1,00$ & \\
Prioridade 2 & 0,99 & 0,$85 ; 1,17$ & $\mathbf{0 , 0 0 2}$ \\
Prioridade 3 & 1 & & $\mathbf{0 , 0 1 3}$ \\
Prioridade 4 & & & \\
Tempo de espera & & & \\
Acima de 6 horas & 0,80 & 0,$70 ; 0,92$ & \\
Até 6 horas & 1 & & \\
Situação de atendimento & & 0,$70 ; 0,95$ & \\
Alta/transferência & 1 & & \\
Óbito & & & \\
\hline
\end{tabular}

RP: Razão de Prevalência; IC 95\%:Intervalo de Confiança de 95\%.

p: teste de associação usando o Qui-quadrado de Wald. Fonte: Autores.

Acerca do perfil sociodemográfico dos pacientes admitidos na UTI adulto do hospital investigado, o mesmo foi observado em estudo realizado em um hospital público de Pernambuco (Mendonça et al., 2019), ao identificar que a maioria das solicitações de leitos de UTI eram provenientes de pacientes do sexo masculino, com idade igual ou superior a 65 anos. Resultado semelhante foi encontrado, onde mais da metade dos pacientes investigados, também era do sexo masculino (Araújo et al., 2020). Esse dado pode estar relacionado ao pouco interesse dos homens, pelo cuidado à saúde, onde muitas vezes só procuram os serviços de saúde, quando o fator de gravidade já está instalado. Além disso, convém destacar que adotam menos hábitos de vida saudáveis, em relação ao público feminino.

Nesta pesquisa, em relação à faixa etária dos pacientes, com solicitação de leitos de UTI, verificou-se que idosos a partir de 65 anos representaram a maior parte da demanda solicitante, corroborando com o estudo de Prece et al. (2016), ao destacar que a média de idade dos participantes foi de 71, 32 anos. Isto pode estar relacionado com o novo perfil epidemiológico de envelhecimento da população, verificada especialmente, em países em desenvolvimento, onde ocorre o aumento da demanda de idosos pelos serviços de saúde. O desenvolvimento de comorbidades que culminam geralmente em internação hospitalar e solicitação de leitos de UTI está atrelado ao processo de envelhecimento, uma vez que implica em declínio das funções do corpo e comprometimento das condições de saúde (Talizin et al., 2019). Outro ponto de vista está 
relacionado às condições sensíveis à assistência ao idoso na Atenção Primária à Saúde (APS), tido como fator preponderante das internações na UTI. Assim, a fragilidade neste nível de atenção, quanto aos problemas de saúde dos idosos, repercute no agravamento dessas condições e, consequentemente, na necessidade de hospitalização (Santos et al., 2019). Embora, o envelhecimento seja algo natural do ciclo da vida e não tenha relação direta com o adoecimento, os idosos estão mais susceptíveis e ocupam maior demanda por hospitalizações.

Ao se analisar a procedência dos pacientes que solicitaram leitos de UTI, o resultado observado é corroborado por Vargas et al. (2020) ao afirmarem que existe discrepância nas cidades brasileiras entre a oferta de leitos no sistema público de saúde e a demanda de atenção à saúde do cidadão. A maior demanda por leitos observada nesta pesquisa foi proveniente dos municípios que compõem a ADS 4, que corresponde ao município de Sobral, região sede do hospital da pesquisa. Pacientes das demais ADS são encaminhados à Sobral, uma vez que, em seus municípios a resolutividade não consegue ser suprida. Para o Conselho Federal de Medicina (2016) o serviço de UTI de cada instituição hospitalar deve priorizar, nos protocolos de admissão e alta, especificamente, o diagnóstico e a necessidade do paciente, bem como, os serviços disponíveis a serem ofertados, que devem estar de acordo com as condições de saúde dos pacientes, independentemente de sua procedência. Deste modo, é possível imaginar que a fragilidade na oferta de leitos de UTI pode estar atrelada a um expressivo impacto financeiro para os cofres públicos, o que inviabiliza a ampliação do número de leitos.

Apesar de não ter sido identificada associação estatística entre os achados diagnósticos dos pacientes solicitantes de leito de UTI com o desfecho, foi possível identificar que o sistema orgânico comprometido reflete diretamente, na gravidade, prognóstico e perfil da necessidade por cuidados intensivos, destacando-se os diagnósticos de origem respiratória, cardiovascular e digestivas, que foram os mais prevalentes. Resultados semelhantes foram encontrados na relação entre os principais diagnósticos de internações em UTI, por causas ligadas aos sistemas circulatório e respiratório (Bonfada et al., 2020). Acredita-se que conhecer os tipos de agravos mais frequentes pode subsidiar a equipe, para planejar ações de educação permanente, aquisição de tecnologias e a adaptação da estrutura das unidades, para melhorar a capacidade de atendimento.

No que concerne à prioridade de atendimento, os pacientes que conseguiram a vaga na UTI tiveram maior ocorrência relativa à prioridade de atendimento 1. Isto está de acordo com Caldeira et al. (2010), que defendem a importância de se admitirem pacientes em UTI, com prioridades 1 e 2, em relação àqueles com prioridades 3,4 e 5 , pois ressaltam a necessidade de se categorizar os pacientes, para utilização dos recursos disponíveis, de forma mais eficiente. Os dilemas envolvendo a admissão dos pacientes nas UTI, consideram as grandes demandas por suportes existentes apenas neste setor, bem como a restrição de leitos (White, Cardenas \& Nates, 2017). A determinação de critérios para as admissões na UTI é de suma importância para viabilizar uma assistência adequada, apesar de ser tênue a linha entre a alocação correta de cada paciente e o acesso isonômico.

Quanto ao tempo de espera, a maior parte das solicitações foi superior a seis horas, para obter vaga no leito de UTI. Segundo Konder e O'dwyer (2019), pacientes em estado crítico, que permanecem aguardando vaga na UTI, com atraso de mais de quatro horas, aumentam o tempo de permanência hospitalar e têm maior risco de mortalidade, além de maiores custos para o sistema de saúde. Políticas de regulação e gerenciamento das demandas de pacientes graves, equiparando os recursos disponíveis em tempo hábil, são propostas a fim de reduzir o tempo prolongado de espera por leito de UTI (Goldwasser et al., 2018). Assim, considera-se importante realizar avaliação dos seguintes fatores relacionados ao tempo de espera: resolução da solicitação, investigação dos eventos adversos associados à demora para conseguir o leito e potencialização dos serviços dos níveis de atenção primária e secundária, a fim de subsidiar o setor terciário.

A quantidade de solicitações de leitos de UTI demonstrou que a maioria dos pacientes teve até duas solicitações. O mesmo foi referido por Konder e O'dweyr (2019) em que a maioria das solicitações apresentou o leito disponibilizado após 24 horas, atendendo a 48,7\% das solicitações de 24 a 96 horas. Apenas 30,8\% das solicitações são atendidas nas primeiras 24 
horas. O número de óbitos se eleva proporcionalmente ao tempo de espera por leito de UTI. Para cada hora esperada, o risco de óbito aumenta em 1,5\%. Tempo de espera superior a 12 horas mostrou uma taxa de óbito de $46 \%$ e, subiu para $57 \%$, quando o tempo de espera excedeu 24 horas (Cardoso et al., 2011). Possivelmente, esses pacientes já chegaram à unidade hospitalar em estado crítico. A eficiência dos processos regulatórios e o acesso à vaga no leito de UTI, mediante a primeira solicitação, poderá minimizar as complicações do paciente e, consequentemente, o número de óbitos.

Embora, as solicitações de leito de UTI tenham sido resolvidas em sua maioria, a prevalência de óbitos dentre os pacientes que não conseguiram a vaga na UTI foi elevada. Este fato por estar relacionado ao tempo prolongado de espera por leito e ao perfil dos pacientes, em sua maioria idosos. Sousa et al. (2017) referem que a taxa de óbito dos pacientes acima de 60 anos é elevada, especialmente quando associada aos procedimentos invasivos e ao declínio funcional deles. É importante destacar que a identificação precoce e adequada do grau de necessidade clínica do paciente por um leito hospitalar, quer seja em UTI ou outro setor, é essencial para a resolutividade da sua condição de saúde, além de evitar a ocorrência de óbitos.

Uma contribuição deste estudo consistiu em dar informações capazes de auxiliar na gestão hospitalar, bem como, refletir sobre aspectos que negligenciam o acesso dos pacientes aos leitos de UTI e, assim, contribuir para a melhoria da oferta e consequentemente, reduzir os índices de morbimortalidade.

Este estudo apresentou como limitação, a indisponibilidade de alguns dados no setor que não foram completamente preenchidos. Outro aspecto foi a dificuldade de obtenção dos dados devido à diversificação das fontes de coleta de dados.

\section{Conclusão}

A capacidade de atendimento hospitalar foi restrita na resolução dos problemas da população do município de Sobral e adjacências, em relação à terapia intensiva, pois a oferta de leitos foi inferior à demanda de solicitações.

Os estudos desenvolvidos no âmbito da disponibilidade de leitos de UTI, apresentam recortes diversos, devido às especificidades das diferentes localidades, na condução do gerenciamento sobre a distribuição de leitos, conforme às necessidades da população. Assim, sugere-se a realização de novas pesquisas, a fim de consolidar os resultados aqui encontrados.

\section{Referências}

Alves Júnior, M. A., et al. Avaliação do tempo de desocupação e ocupação de leitos como parâmetros de governança em pacientes internados em unidade de terapia intensiva na rede pública (2020). Rev Bras Ter Intensiva, 32 (3), 412-417. https://www.scielo.br/pdf/rbti/v32n3/0103-507X-rbti-32-03-0412.pdf

Angus, D. C, et al. Caring for the critically ill patient. Current and projected workforce requirements for care of the critically ill and patients with pulmonary disease: can we meet the requirements of an aging population? (2000). JAMA, 284 (21), 2762-2770. https://jamanetwork.com/journals/jama/fullarticle/193318

Araújo, A. C. Qet al. Incidentes relacionados à assistência à saúde em idosos hospitalizados (2020). Rev enferm UFPE on line, 14, e244639. https://periodicos.ufpe.br/revistas/revistaenfermagem/article/view/244639/35727

Barros, A. J. D., \& Hirakata, V. N. Alternatives for logistic regression in cross-sectional studies: an empirical comparison of models that directly estimate the prevalence ratio (2003). BMC Med Res Methodol, 3, 21. https://www.ncbi.nlm.nih.gov/pmc/articles/PMC521200/

Bonfada, D., Barbosa, I. C. R., Lima, K. C., \& Garcia-Altes, A. Gasto de internação de idosos em unidades de terapia intensiva nos hospitais privados de uma capital do nordeste brasileiro (2020). Rev bras geriatr gerontol, 23 (2), e200020. https://doi.org/10.1590/1981-22562020023.200020.

Brasil. Resolução ${ }^{\circ}$ 2.271, 14 de fevereiro de 2020. Define as unidades de terapia intensiva e unidades de cuidado intermediário conforme sua complexidade e nível de cuidado, determinando a responsabilidade técnica médica, as responsabilidades éticas, habilitações e atribuições da equipe médica necessária para seu adequado funcionamento. Brasília: Imprensa Nacional. https://www.in.gov.br/en/web/dou/-/resolucao-n-2.271-de-14-de-fevereiro-de-2020-253606068

Caldeira, V. M. H., et al. Critérios para admissão de pacientes na unidade de terapia intensiva e mortalidade (2010). Rev Assoc Med Bras., 56 (5), 528-534. http://www.scielo.br/scielo.php?script=sci_arttext\&pid=S0104-42302010000500012\&lng=en\&nrm=iso

Capela, I. L. B., et al. Fatores associados à alta de idosos internados em uma Unidade de Terapia Intensiva Referência (2021). Saud Pesq, 14 (1), e7833. https://doi.org/10.17765/2176-9206.2021v14n1.e7833

Cardoso, L. T., et al. Impact of delayed admission to intensive care units on mortality of critically ill patients: a cohort study (2011). Crit Care, 15 (1), R28. https://www.ncbi.nlm.nih.gov/pmc/articles/PMC3222064/pdf/cc9975.pdf 
Research, Society and Development, v. 10, n. 8, e2610816572, 2021

(CC BY 4.0) | ISSN 2525-3409 | DOI: http://dx.doi.org/10.33448/rsd-v10i8.16572

Ceará. Secretaria Estadual de Saúde. Portaria no. 2.108/2019. Dispõe sobre aspectos organizativos-operacionais das regiões de saúde, nos termos da lei estadual no 17.006, de 30 de setembro de 2019. Fortaleza-CE: Secretaria Estadual de Saúde, 2019a.

Chagas, F. A. F. R., et al. As tutelas de urgência com pedidos de saúde em sede de plantão judiciário noturno (2019). Saúde em Debate, 43 (4), $232-243$. https://www.scielo.br/pdf/sdeb/v43nspe4/0103-1104-sdeb-43-spe04-0232.pdf

Conselho Federal de Medicina. Resolução CFM No 2.156/2016. Estabelece os critérios de admissão e alta em unidade de terapia intensiva. Disponível em: https://sistemas.cfm.org.br/normas/visualizar/resolucoes/BR/2016/2156

Cotrim Junior, D. F., \& Cabral, L. M. S. Crescimento dos leitos de UTI no país durante a pandemia de Covid-19: desigualdades entre o público x privado e iniquidades regionais (2020). Physis: Revista de Saúde Coletiva, 30 (3), e300317. https://www.scielosp.org/pdf/physis/2020.v30n3/e300317/pt.

Coutinho, L. M. S., Scazufca, M., Menezes, P. R. Methods for estimating prevalence ratios in crosssectional studies (2008). Rev Saúde Pública, 42 (6), $992-$ 998. https://www.scielo.br/pdf/rsp/v42n6/en_7118.pdf

Goldwasser, R. S., et al. Planejamento e compreensão da rede de terapia intensiva no Estado do Rio de Janeiro: um problema social complexo (2018). Rev bras ter intensiva, 30 (3), 347-357. http://www.scielo.br/scielo.php?script=sci_arttext\&pid=S0103-507X2018000300347\&lng=en\&nrm=iso

Goldwasser, R. S., et al. Difficulties in access and estimates of public beds in intensive care units in the state of Rio de Janeiro (2016). Rev Saúde Pública, 50 (19), 1-10. http://www.scielo.br/scielo.php?script=sci_arttext\&pid=S0034-89102016000100401\&lng=en\&nrm=iso

Instituto de Saúde e Gestão Hospitalar (ISGH). Disponível em: https://www.isgh.org.br/hospital-regional-norte

Konder, M., \&e O'dwyer, G. Unidades de Pronto Atendimento como unidades de internação: fenômenos do fluxo assistencial na rede de urgências (2019). Physis: Revista de Saúde Coletiva, 29 (02), e290203. https://doi.org/10.1590/S0103-73312019290203

Mendonca, J. G., et al. Perfil das internações em Unidades de Terapia Intensiva Pediátrica do Sistema Único de Saúde no estado de Pernambuco, Brasil (2019). Ciênc saúde coletiva, 24 (3), 907-916. http://www.scielo.br/scielo.php?script=sci_arttext\&pid=S1413-81232019000300907\&lng=pt\&nrm=isov

Nates, J. L., et al. ICU admission, discharge, and triage guidelines: a framework to enhance clinical operations, development of institutional policies, and further research (2016). Crit Care Med., 44 (8), 1553-602. https://pubmed.ncbi.nlm.nih.gov/27428118/ .

Prece A., et al. Perfil de Pacientes em Terapia Intensiva: Necessidade do Conhecimento para Organização do Cuidado (2017). Cad da Esc de Saúde, 2 (16), 35-48. https://portaldeperiodicos.unibrasil.com.br/index.php/cadernossaude/article/view/2462

Santos, F. R. Q., et al. Resultados adversos de admissões tardias à unidade de terapia intensiva a partir do pronto-socorro (2020). Rev bras ter intensiva, 32 (1), 92-98. http://www.scielo.br/scielo.php?script=sci_arttext\&pid=S0103-507X2020000100092\&lng=en\&nrm=iso

Santos, K. M R., et al. Internações por condições sensíveis à atenção primária à saúde em população idosa no estado do Rio Grande do Norte, Brasil, no período de 2008 a 2016 (2019). Rev. Bras Geriatr Gerontol, 22 (4), e180204. https://www.scielo.br/pdf/rbgg/v22n4/pt_1809-9823-rbgg-22-04-e180204.pdf

Sousa, A. F. L., et al. Óbitos em idosos com infecção adquirida em Unidades de Terapia Intensiva (2017). Rev Bras Enferm, 70 (4), $733-739$. http://www.scielo.br/scielo.php?script=sci_arttext\&pid=S0034-71672017000400733\&lng=en\&nrm=iso

Talizin, T. B., et al. Risk factors for mortality in geriatric patients admitted to the intensive care unit at a public hospital (2019). Geriatr Gerontol Aging, 13, 69-74. https://cdn.publisher.gn1.link/ggaging.com/pdf/en_v13n2a03.pdf

Vargas, M. A. O., et al. Gestão da disponibilidade de leitos na terapia intensiva no contexto da internação por ordem judicial (2020). Rev Latino-Am Enfermagem, 28, e3271. http://www.scielo.br/scielo.php?script=sci_arttext\&pid=S0104-11692020000100329\&lng=en\&nrm=iso

White, S. T., et al. O que todo intensivista deve saber sobre critérios de admissão à unidade de terapia intensiva (2017). Rev bras ter intensiva, 29 (4), $414-417$. http://www.scielo.br/scielo.php?script=sci_arttext\&pid=S0103-507X2017000400414\&lng=en\&nrm=iso 